\title{
Erratum: Increased spread and replication efficiency of Listeria monocytogenes in organotypic brain-slices is related to multilocus variable number of tandem repeat analysis (MLVA) complex
}

Claudia Guldimann ${ }^{1,2}$, Michelle Bärtschi ${ }^{1}$, Joachim Frey ${ }^{3}$, Andreas Zurbriggen ${ }^{1}$, Torsten Seuberlich ${ }^{1}$ and Anna Oevermann ${ }^{1 *}$

\section{Erratum}

The original version of this article unfortunately contained a mistake. Figures two, three and four (Figs. 1, 2 and 3 here, respectively) and their associated legends were interchanged in the HTML and PDF versions of this manuscript. The correct versions are given below. In addition, Figure Five (Fig. 4 here) was missing in the HTML version of this manuscript. The correct figure Five (Fig. 4 here) is also included below.

Published online: 03 September 2015

\section{Author details}

'Division of Neurological Sciences, Neurocenter, Department of Clinical Research and Veterinary Public Health, Vetsuisse Faculty, University of Bern, Bern, Switzerland. ${ }^{2}$ Graduate school for Cellular and Biomedical Sciences, University of Bern, Bern, Switzerland. ${ }^{3}$ Institute of Veterinary Bacteriology, Vetsuisse Faculty, University of Bern, Bern, Switzerland.

\footnotetext{
* Correspondence: anna.oevermann@vetsuisse.unibe.ch

${ }^{1}$ Division of Neurological Sciences, Neurocenter, Department of Clinical Research and Veterinary Public Health, Vetsuisse Faculty, University of Bern, Bern, Switzerland
} 


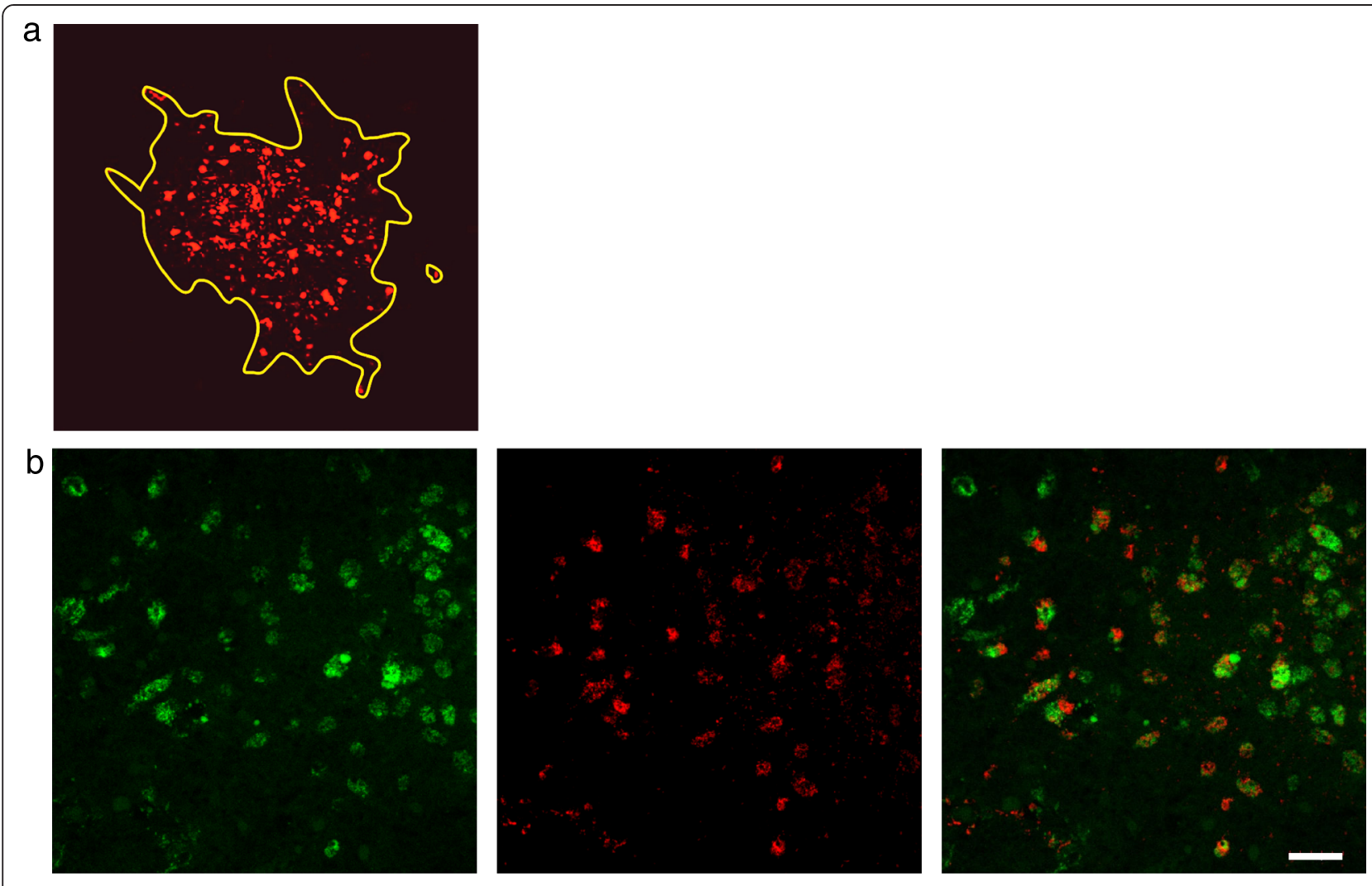

Fig. 1 Immunofluorescence stained confocal images of bacteria in infected brain-slices. a: Delineation of an infection focus (yellow line). L. monocytogenes are stained in red. The surface area covered by L. monocytogenes was drawn and calculated using the Fluoview software (Olympus FV10-ASW Version 03.01.01.09) Magnification 20x. b: Representative double-immunofluorescence of a L. monocytogenes infected brain-slice. The vast majority of bacteria are found within microglia. Left: Microglia are stained with CD68 in green. Center: L. monocytogenes in red. Right: Overlay (bar $=40 \mu \mathrm{m})$ 


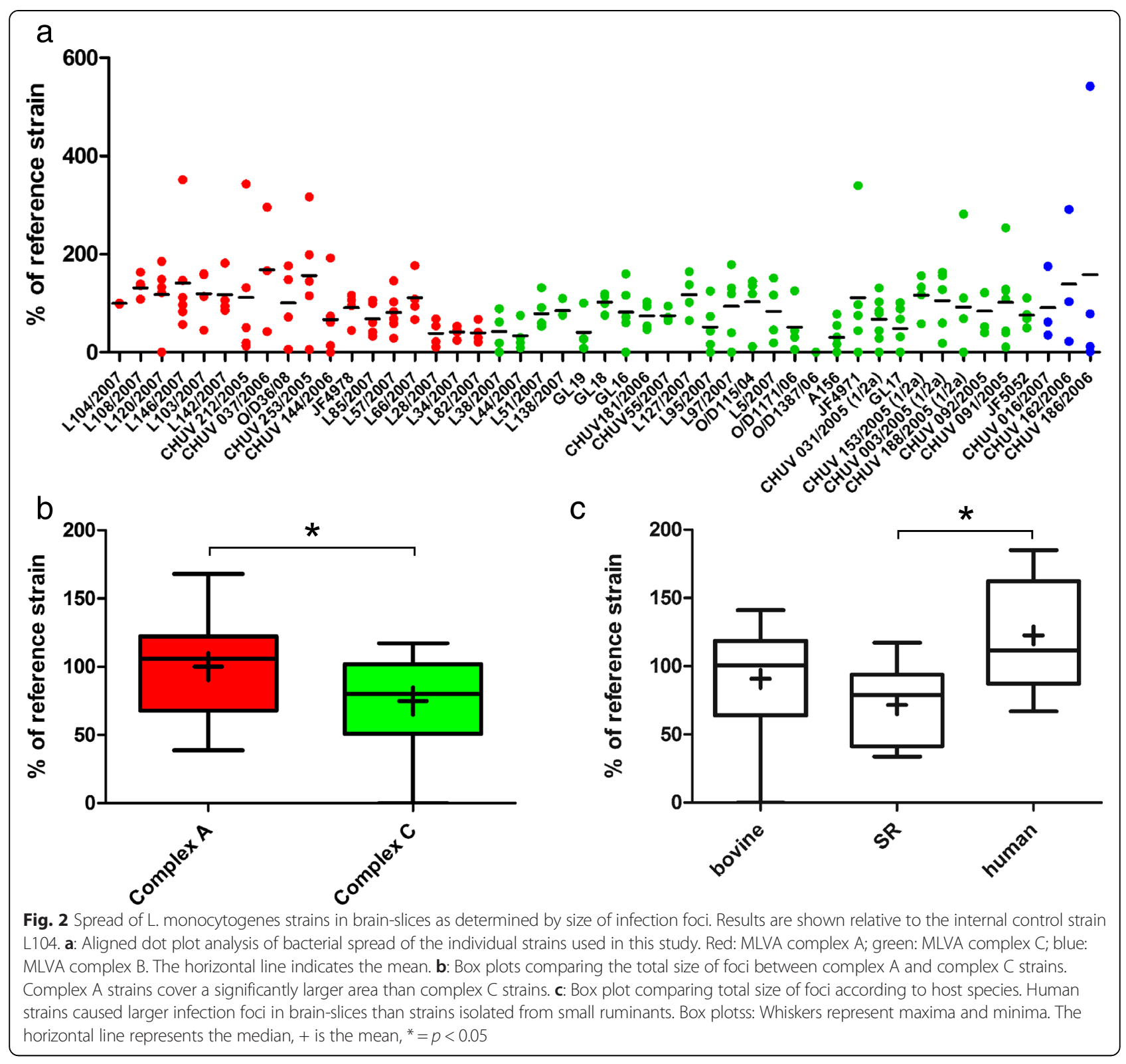




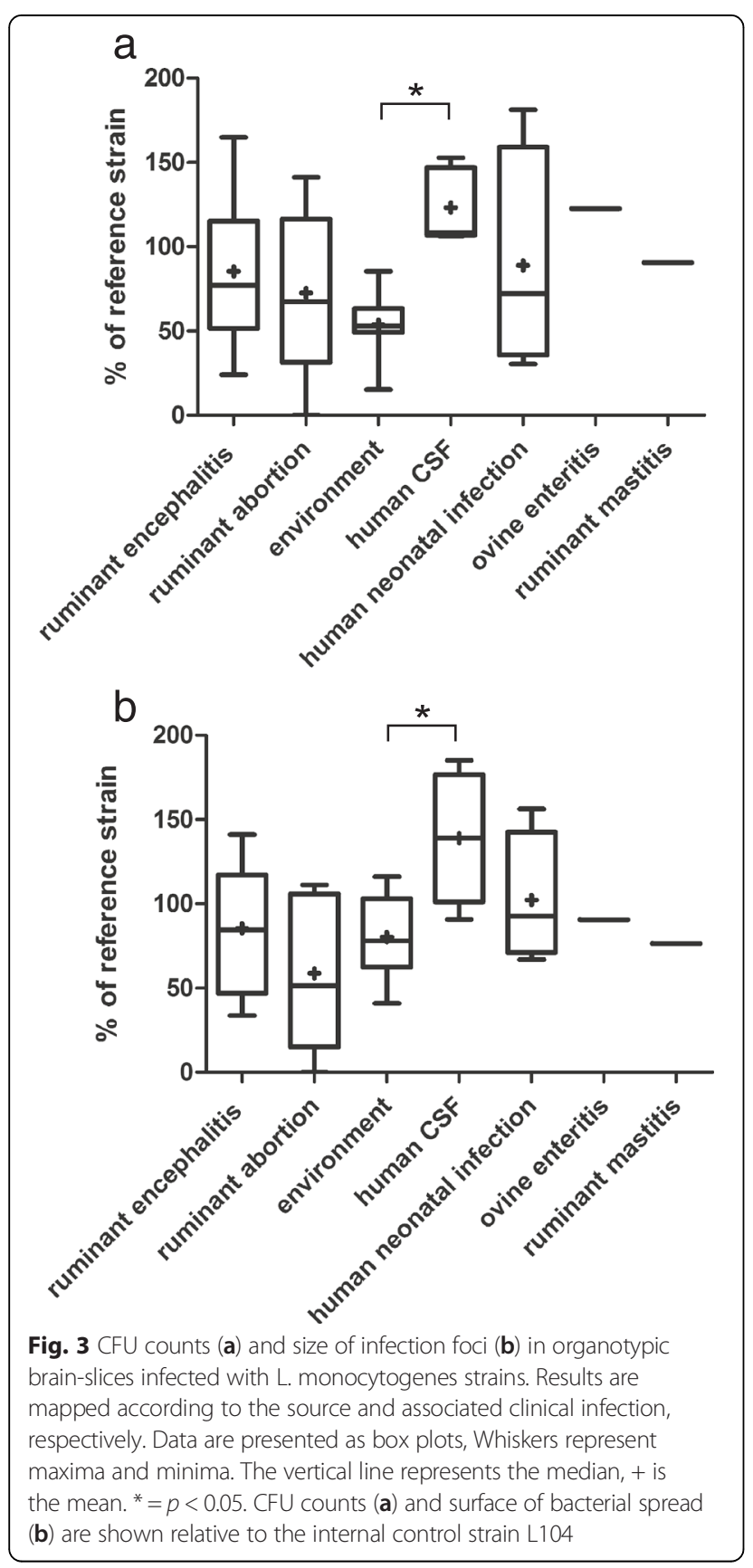




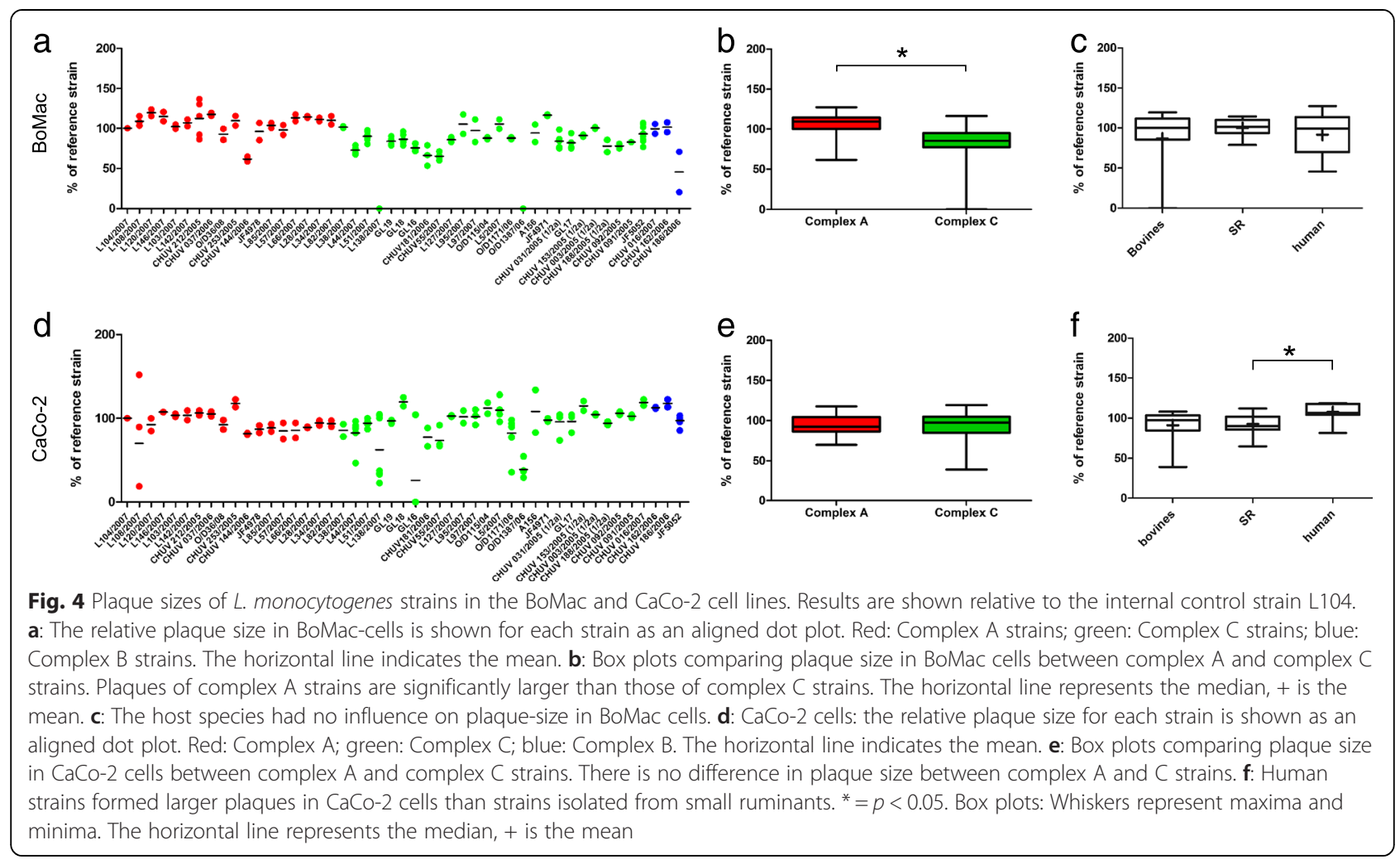

